Rev. Biol. Trop., 47(4): 775-784, 1999

www.ucr.ac.cr www.ots.ac.cr www.ots.duke.edu

\title{
Efectos de plantaciones abandonadas de especies exóticas y nativas sobre la regeneración natural de un bosque montano en Colombia
}

\author{
Jaime Cavelier y Carolina Santos
}

Departamento de Ciencias Biológicas, Universidad de los Andes, AA 4976, Bogotá, Colombia. Fax: 57-1-2841890.

Correo electrónico:jcavelie@zeus.uniandes.edu.co

Recibido 22-X-1998. Corregido 20-IV-1999. Aceptado 10-V-1999

\begin{abstract}
Vegetation surveys were carried out during 1994 in 0.1. ha plots in abandoned plantations of Pinus radiata, Cupressus lusitanica, Eucalyptus globulus, Alnus acuminata and in a secondary upper montane rain forest in the Central Andes of Colombia. The regeneration forest had the higher number of plant species (33) followed by the E. globulus (26) and A. acuminata (16) plantations. Abandoned plantations of $P$. radiata and $C$. lusitanica, had only three species. There were only eleven species in common between the regeneration forest and the plantation of E. globulus (Baccharis latifolia, Cordia cylindrostachya, Dunalia solanacea, Leandra melanodesma, Lippia hirsuta, Monnina angustata, Solanum aff. scorpioideum, S. aphydendron, Sphaeropteris quindiuensis, Tobouchina mollis and Verbesina nudipes), and only seven when compared to the A. acuminata plantation (Abatia parviflora, Asploddianthus pseudostuebelli, Bocconia frutescens, Leandra melanodesma, Lippia hirsuta y Solanum aff. scorpioideum y Verbesina nudipes). With the exception of Cordia cylindrostachya, Bocconia frutescens and Tibouchina mollis, all other species are understory shrubs dispersed by wind or birds. In the understory of the $P$. radiata plantation there was abundant regeneration of the Colombian national tree, Ceroxylon quindiuensis. Tree height and basal area were significantly higher in the $P$. radiata and $C$. lusitanica plantations than in the regeneration forest. Of the environmental and biological variables measured in this study, the accumulation of needles under $P$. radiata and $C$. lusitanica plantations, and the high biomass of fine roots under the $C$. lusitanica plantation, could be the main limiting factors for the establishment of a higher number of species of the native forest. The chemical properties of the soils varied greatly, and there were no consistent differences between the soils under exotic and native species.
\end{abstract}

Key words: Alnus, Central Andes, Eucalyptus, Pinus, plantations, regeneration, tropical montane forest.

Los bosques montanos tropicales en la zona andina han sufrido una presión de deforestación mayor que los bosques húmedos de tierras bajas a causa de los patrones de colonización (Molano 1992, Bedoya \& Klein 1996, Young 1996). En Colombia, el proceso de deforestación ha reducido las áreas de bosque montano entre un $73 \%$ (Cavelier \& Etter 1995) y un $90 \%$ (Henderson et al. 1991). Como parte de este proceso, la mayoría de los bosques maduros montanos en Colombia, en particular los bosques alto andinos (ca. 2500ca. 3200 m.s.n.m), fueron utilizados a partir de la década de los años 50 para la producción de carbón vegetal y posteriormente para la adecuación de pastizales para la ganadería de altura (Londoño 1994). Este proceso ocurrió en forma paralela en los bosques del piedemonte andino (300-800 m.s.n.m) (Viña \& Cavelier 1999) y en los bosques montanos bajos (ca. 1200- ca. 2500 m.s.n.m) donde el principal uso de la tierra después de la deforestación fue agrícola (v.g. café) y no ganadero. En algunas áreas de pastizales, a finales de la década de los años 60, fueron establecidas plantaciones de especies exóticas (Pinus radiata, Cupressus 
lusitanica, Eucalyptus globulus) y nativas (Alnus acuminata, Acacia sp.), que tenían como fin la protección de las fuentes de agua. En otras áreas estos pastizales simplemente fueron abandonados, y en ellos se desarrolló un bosque secundario gracias a la lluvia de semillas desde los fragmentos de bosques que quedaron a lo largo de quebradas y en lugares de difícil acceso. Estos bosques secundarios, a pesar de tener un número menor de especies que los bosques maduros, obtienen altos valores de biomasa y producción de hojarasca en poco tiempo (Brown \& Lugo 1990), proporcionando variedad de servicios ambientales (Ewel 1980).

El reemplazo de bosques nativos tropicales por plantaciones, bien sean de carácter productivo o con fines de conservación-protección, ha generado tres principales debates: el efecto sobre la biodiversidad, los suelos y los recursos hídricos. El efecto sobre la riqueza y diversidad de especies, así como sobre los suelos, es generalmente negativo (Savill \& Evans 1986, Sawyer 1993). Sin embargo, estas plantaciones pueden tener efectos neutros (Cavelier \& Tobler 1998) o positivos en el suelo, cuando se usan en programas de rehabilitación de áreas severamente degradadas (Lugo 1992a, Parrota 1992). Cuando se comparan plantaciones y pastizales con relación al rendimiento hídrico de las cuencas, el efecto puede ser positivo, negativo o neutro dependiendo de las condiciones ambientales locales (Kelliher et al. 1993, Waterloo 1994).

En el presente trabajo se comparan la riqueza, diversidad y algunas características fisicoquímicas de los suelos en plantaciones de especies exóticas y nativas, así como en bosques secundarios en el flanco occidental de la Cordillera Central de los Andes colombianos (ca. 2750 m.s.n.m.).

\section{MATERIALES Y MÉTODOS}

Este trabajo fue realizado en la Finca La Picota de propiedad de la Corporación Autónoma Regional del Quindío (CRQ). La Finca está localizada a una altitud media de 2750 m.s.n.m. en áreas que estuvieron cubiertas de selvas sub-andinas (Londoño 1994) y que fueron convertidas, a mediados de este siglo, a pastizales de "kikuyo" (Pennisetum clandestinum) para la explotación ganadera. Esta finca fue incorporada a una amplia zona protectora y de reserva forestal por medio de la Ley 23 de 1963. En los terrenos de la finca fueron establecidas entre 1968 y 1970, plantaciones contiguas de Alnus acuminata, Pinus radiata, $\mathrm{Cu}$ pressus lusitanica y Eucalyptus globulus, con áreas entre 5-15 ha. Estas áreas, con pendientes entre $20 \%$ y $30 \%$ y de topografía ondulada, fueron utilizadas anteriormente como potreros. Las plántulas con que se iniciaron estas plantaciones fueron sembradas a raíz desnuda, a una distancia de $2.5 \mathrm{~m} \times 2.5 \mathrm{~m}$. y en "platos" de un metro de diámetro en donde se removieron los pastos con azadón y machete. Estas plantaciones fueron establecidas con fines de protección de la cuenca del Río Quindío, por lo cual se abandonaron después de su establecimiento. Algunos de los potreros que no fueron sembrados también se abandonaron y actualmente se encuentran como bosques de regeneración natural.

En octubre de 1994 se establecieron parcelas de 0.1 hectáreas ( 10 subparcelas contiguas de $10 \mathrm{~m} \times 10 \mathrm{~m}$ ) en cada una de las plantaciones de 26 años de Alnus acuminata, Pinus radiata, $\mathrm{Cu}$ pressus lusitanica y Eucalyptus globulus, así como en un bosque de regeneración natural de la misma edad. En cada una de las parcelas se tomó una muestra botánica y se midió la altura y el diámetro de todos los individuos con un diámetro a la altura del pecho (d.a.p.) mayor a $2.5 \mathrm{~cm}$. Adicionalmente se tomaron tres muestras al azar de biomasa de raíces y hojarasca (o acículas en el caso de las coníferas). Estas muestras fueron tomadas con un barreno cilíndrico de $5.8 \mathrm{~cm}$ de diámetro por $20 \mathrm{~cm}$ de profundidad. Además, se tomaron al azar tres muestras de suelo en las parcelas de cada plantación y en un potrero control de Pennisetum clandestinum utilizado para el pastoreo de vacas y animales de carga. Los análisis de suelos fueron realizados en el Laboratorio del Instituto Geográfico Agustín Codazzi, Bogotá y los métodos están descritos en Cavelier (1992) y Cavelier \& Tobler (1998). Las muestras 
botánicas fueron identificadas hasta género o especie mediante la comparación del material botánico con las colecciones del Herbario de la Fundación Herencia Verde, Salento, Quindío. La información sobre la presencia de especies en cada una de las subparcelas fue utilizada para la construcción de una curva especies-área en cada una de las plantaciones y en el bosque de regeneración natural, iterando diez veces cada curva y mediante un proceso aleatorio de selección de parcelas. El número de troncos por sub-parcela, diámetro de los árboles, biomasa de hojarasca y de raíces finas $(<5 \mathrm{~mm})$ fueron comparadas entre los diferentes tipos de vegetación mediante un análisis de KruskalWallis. Cuando se presentaron diferencias estadísticamente significativas en alguna de estas variables se usó un análisis de Turkey ( $a$ posteriori) para ver cuales de los tipos de vegetación eran diferentes entre sí.

\section{RESULTADOS}

Estructura: La altura media del dosel, incluidas las especies plantadas, fue mayor en las plantaciones (9.8 -19.3 m) que en el bosque de regeneración natural $(6.5 \mathrm{~m})$ (Cuadro 1$)$. La densidad media de troncos mayores de $2.5 \mathrm{~cm}$ d.a.p. fue mayor en el bosque de regeneración natural y en la plantación de eucalipto, que en las plantaciones de alisos y coníferas. El área basal fue mayor en las plantaciones de coníferas que en las de alisos, eucaliptos o en el bosque de regeneración natural (Cuadro 1). La biomasa de raíces finas fue mayor en la plantación de $C$. lusitanica y la acumulación de hojarasca (acículas) mayor en las plantaciones abandonadas de coníferas (Cuadro 1). La similaridad de las características estructurales entre los diferentes tipos de vegetación están resumidas en el Cuadro 2.

\section{CUADRO 1}

Características florísticas y estructurales de las plantaciones abandonadas, regeneración natural y pastizales activos

$\begin{array}{lcccccc} & \text { Pino } & \text { Ciprés } & \text { Aliso } & \text { Eucalipto } & \text { R.Natural } & \text { Pastos } \\ \text { No. Especies } & & & & & & \\ \text { No. Familias } & 3 & 3 & 16 & 26 & 33 & \\ \text { Altura del dosel }(\mathrm{m}) & 3 & 3 & 10 & 14 & 15 & \\ \text { Area basal }\left(\mathrm{m}^{2} / \mathrm{ha}\right) & 19.33(1.17) & 14.96(0.73) & 11.78(0.97) & 9.89(0.79) & 6.55(0.31) & \\ \text { Densidad }(\mathrm{No} . / \text { área }) & 80.9(1.12) & 53.7(4.8) & 26.52(4.2) & 18.05(1.55) & 14.91(1.27) & \\ \text { Biomasa de raíces }\left(\mathrm{kg} / \mathrm{m}^{2}\right) & 9.30(1.18) & 13.80(1.76) & 10.80(1.02) & 17.60(2.10) & 17.10(1.59) & \\ \text { Biomasa de hojarasca }\left(\mathrm{kg} / \mathrm{m}^{2}\right) & 0.21(0.06) & 1.53(0.25) & 0.16(0.04) & 0.17(0.06) & 0.72(0.07) & 0.89(0.22) \\ & 3.30(0.45) & 2.01(0.33) & 1.48(0.43) & 0.56(0.16) & 0.74(0.11) & 1.03(0.30)\end{array}$

Los valores son los promedios obtenidos de 10 parcelas de $10 \times 10 \mathrm{~m}$ (+/- $1 \mathrm{ES})$. Los valores de biomasa de raíces y hojarasca son el promedio de 3 muestras (+/-1 ES).

\section{CUADRO 2}

Resultados del análisis a posteriori del test de Kruskall-Wallis de las variables estructurales de las plantaciones de pino $(P)$, Ciprés $(C)$, Aliso $(A)$, Eucalipto $(E)$ y Regeneración natural $(R)$

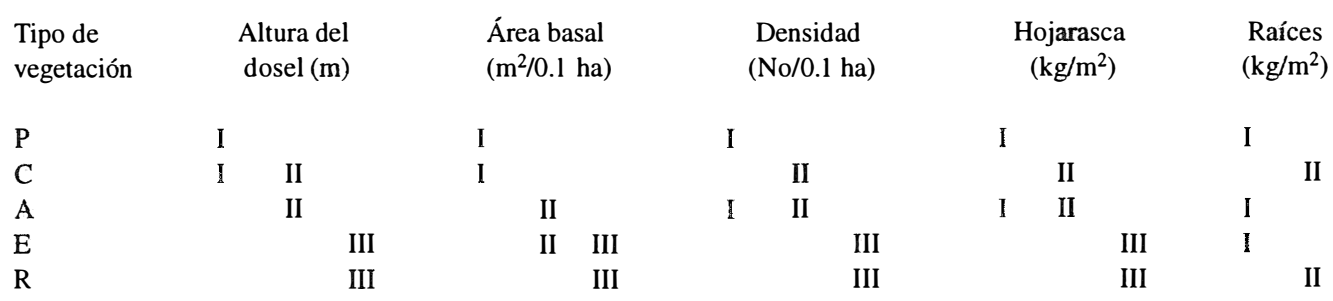

Los números I, II y III agrupan los tipos de vegetación que son similares entre sí ( $\mathrm{P}<0.05)$. 


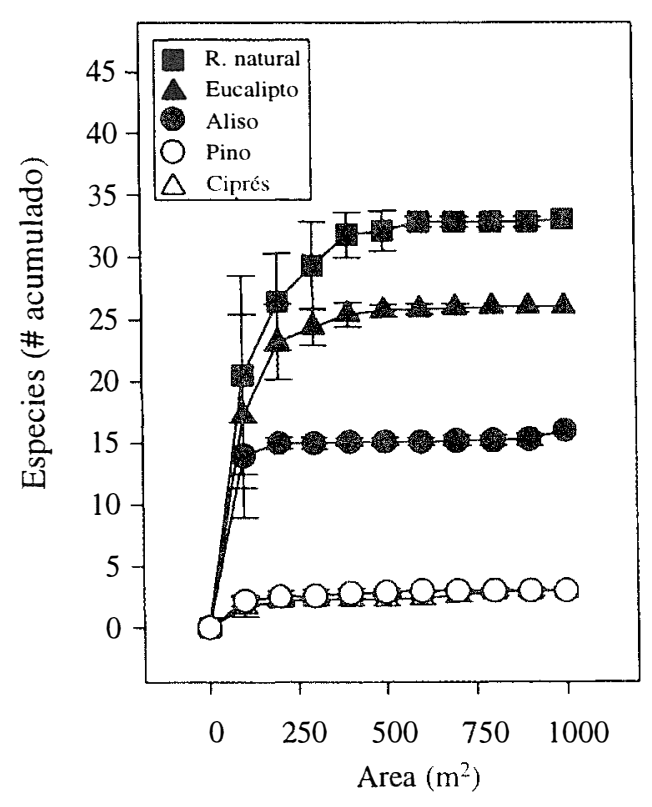

Fig. 1. Curvas de acumulación de especies en las plantaciones y en el bosque de regeneración natural. Cada punto es el valor medio de diez iteraciones y la línea vertical la desviación típica.

Florística: En parcelas de 0.1 ha. el bosque con mayor número de especies leñosas de más de $2.5 \mathrm{~cm}$ d.a.p. fue el bosque de regeneración natural (33 especies) seguido de las plantaciones abandonadas de E. globulus (26) y A. acuminata (16). Las plantaciones de $P$. radiata y $C$. lusitanica sólo tuvieron 3 especies incluida la especie plantada (Fig. 1). La regeneración de la vegetación nativa que crece a la sombra de las plantaciones abandonadas de alisos y eucaliptos, contiene un número menor de especies que el bosque de regeneración natural y pocas especies en común con este. Así, las plantaciones de eucalipto, aliso y el bosque de regeneración natural sólo tienen cuatro especies en común (Leandra melanodesma y Solanum aff. scorpioideum dispersadas por aves, Lippia hirsuta y Verbesina nudipes, dispersadas por viento). Además de estas cuatro especies, el bosque de regeneración natural, comparte otras siete especies con las plantaciones de eucalipto (Baccharis latifolia, Cordia cylindrostachya, Dunalia solanacea, Monnina angustata, Solanum aphydendron, Sphaeropteris quindiuensis, Tibouchina mollis), y tres con las plantaciónes de aliso (Abatia parviflora, Asploddianthus pseudostuebelli y Bocconia frutescens) (Anexo 1). Con la posible excepción de Cordia cylindrostachya, Bocconia frutescens y Tibouchina mollis, las cuales son árboles pequeños, todas las demás tienen hábitos arbustivos. En la plantación de pinos se encontró regeneración del árbol nacional de Colombia, la palma Ceroxylon quindiuensis.

Suelos: Los suelos de las plantaciones abandonadas, del bosque de regeneración natural y del potrero utilizado como control, mostraron similitud en la textura (de franco a franco-arenosa) y una gran variabilidad en las características químicas (Cuadro 3). De acuerdo a criterios agronómicos (Landon 1991), la capacidad de intercambio catiónico varió de media a alta, con los valores más bajos en el bosque de regeneración, lo cual es resultado, por lo menos parcialmente, de los bajos porcentajes de carbono. En la plantación de ciprés se observó el valor más bajo de bases totales, de saturación y de calcio intercambiable. El pH de los suelos varió de bajo a medio, con los valores menores en las plantaciones de aliso y ciprés. Estos valores bajos están correlacionados con las mayores concentraciones de aluminio intercambiable. Los valores de fósforo disponible (Bray II), fueron bajos con la excepción de las plantaciones de aliso y de eucalipto. Los suelos de los pastizales activos tuvieron los valores más altos de capacidad de intercambio catiónico. En general, los suelos de las plantaciones, bosque de regeneración natural y potreros, pueden considerarse de fertilidad moderada a alta.

\section{DISCUSIÓN}

Los resultados de este estudio están basados en la comparación de parcelas de 0.1 ha. A pesar de la falta de réplicas verdaderas (cada parcela fue subdividida en 10 subparcelas de 10 m. x $10 \mathrm{~m}$.), el uso de parcelas únicas de $0.1 \mathrm{a}$ 1.0 ha. han sido útiles cuando se comparan diferentes plantaciones, bosques secundarios 


\section{CUADRO 3}

Características fisicoquímicas de los suelos de las plantaciones abandonadas, regeneración natural y pastizales activos.

\begin{tabular}{|c|c|c|c|c|c|c|}
\hline & Pino & Ciprés & Aliso & Eucalipto & R.Natural & Pastizales \\
\hline Arena \% & $58(3.05)$ & $62(1.5)$ & $58.66(3.52)$ & $53.33(1.33)$ & $55.33(1.33)$ & $64.66(1.33)$ \\
\hline Limos \% & $29.33(4.8)$ & $28(1.15)$ & $25.33(1.76)$ & $30(0.0)$ & $32(1.76)$ & $64.66(1.33)$ \\
\hline Arcilla \% & $2.66(2.90)$ & 10 & $16(2.0)$ & $16.66(1.33)$ & $13.33(0.66)$ & $9.33(1.33)$ \\
\hline $\mathrm{pH}$ & $5.53(0.08)$ & $5.06(0.06)$ & $4.7(0.32)$ & $5.76(0.38)$ & $5.6(0.17)$ & $5.76(0.26)$ \\
\hline $\mathrm{Al}(\mathrm{me} / \mathrm{l00 \textrm {g } )}$ & 0.2 & $1.2(0.35)$ & $0.76(0.52)$ & 0.2 & 1.3 & 0.4 \\
\hline C.I.C. & $\begin{array}{l}32.3(3.71) \\
(\mathrm{me} / 100 \mathrm{~g})\end{array}$ & $37.26(3.03)$ & $28.13(3.69)$ & $23.93(0.96)$ & $16.2(2.13)$ & $40.7(3.04)$ \\
\hline Bases totales & $8.43(1.92)$ & $2.54(0.92)$ & $7.9(0.38)$ & $14.86(1.75)$ & $6.23(2.04)$ & $10.93(2.3)$ \\
\hline $\mathrm{Ca}(\mathrm{me} / 100 \mathrm{~g})$ & $5.73(1.73)$ & $2.13(0.69)$ & $4.5(0.3)$ & $9.46(1.21)$ & $3.33(1.18)$ & $10.8(1.43)$ \\
\hline $\mathrm{Mg}$ & $2.3(0.17)$ & $2.13(0.35)$ & $2.4(0.61)$ & $3.9(0.4)$ & $1.86(0.48)$ & $2.4(0.13)$ \\
\hline$(\mathrm{me} / \operatorname{lo0g}($ & $036(0.03)$ & $0.46(0.16)$ & $1(0.057)$ & $1.46(0.36)$ & $0.93(0.38)$ & $0.93(0.12)$ \\
\hline $\mathrm{K}(\mathrm{me} / 100 \mathrm{~g})$ & $0.033(0.008)$ & $0.083(0.016)$ & $0.0023(0.003)$ & $0.053(0.023)$ & $0.08(0.02)$ & $0.036(0.003)$ \\
\hline $\mathrm{Na}(\mathrm{me} / 100 \mathrm{~g})$ & $25.46(3.04)$ & $12.96(2.17)$ & $29.36(4.83)$ & $62.9(9.56)$ & $36.6(6.92)$ & $28.03(7.8)$ \\
\hline Sat. Bases & $4.29(0.67)$ & $5.13(0.1)$ & $4.35(0.67)$ & $3.35(0.47)$ & $3.13(0.33)$ & $4.89(0.32)$ \\
\hline $\begin{array}{l}\text { C \% } \\
P(p p m)\end{array}$ & $2.66(1.66)$ & $5(0.57)$ & $19.66(10.68)$ & $15.33(3.6)$ & $8(1.52)$ & $4.66(0.66)$ \\
\hline
\end{tabular}

(Lugo 1992b, Cavelier \& Tobler 1998) y bosques montanos tropicales de tierras bajas y altas (Gentry 1988, 1992, Clinebell et al. 1995).

Estructura y florística del bosque de regeneración natural y plantaciones: En el bosque de regeneración natural se encontraron 33 especies de plantas leñosas con d.a.p. $>2.5$ $\mathrm{cm}$. Este número de especies es menor al que se encontraría en un bosque primario a esta misma altitud (ca. 53 especies; Gentry 1992). Las especies más comunes en este tipo de vegetación fueron las Melastomataceae Miconia pustulata (39\% de los individuos) y Axinea macrophyla (9\%), las cuales tienden a formar bosques homogéneos durante los estadios sucesionales tempranos (Stiles \& Roselli 1993, Bello \& Katib 1995). Durante el trabajo de campo fue posible observar una cohorte de in- dividuos muertos de otra especie de Melastomataceae, que había sido reemplazada por cohortes de estas dos especies. Además de las Melastomataceae (54\% con 7 especies), las dos familias de plantas con mayor número de individuos fueron Solanaceae (12\% con 6 especies) y Asteraceae (10\% con 3 especies).

En la plantación de $E$. globulus, se encontraron 26 especies nativas, y sólo 11 en común con el bosque de regeneración natural, de las cuales cuatro especies se comparten también con la plantacion de aliso. El valor relativamente alto de especies en esta plantación puede atribuirse a un dosel relativamente bajo (ca. $9 \mathrm{~m}$ ), y a un área basal más parecida a la del bosque de regeneración natural que a la de las plantaciones de pino, ciprés y aliso (Cuadro 2). En la plantación de Eucalipto no parecen expresarse los efectos alelopáticos que han sido 
reportados en otras plantaciones (Lima 1987), probablemente por la acción de las altas precipitaciones y la baja densidad de la plantación. Adicionalmente, los suelos no presentaron mayores diferencias con relación al bosque de regeneración natural y la biomasa de raíces y hojarasca no limitan el establecimiento de plántulas de especies nativas como sí parece ser el caso en las plantaciones abandonadas de $P$. $r a$ diata y $C$. lusitanica.

En la plantación abandonada de Alnus acuminata, se encontraron 16 especies de plantas en la regeneración natural, 10 menos que en la plantación de E. globulus y 17 menos que en el bosque de regeneración natural. La presencia de un número menor de especies nativas en el sotobosque de plantaciones abandonadas de alisos en comparación con bosques secundarios de la misma edad parece ser una constante, como lo demuestran otros dos estudios en la misma cordillera. A 3100 m.s.n.m. se encontraron 26 especies nativas en la plantación y 33 en el bosque secundario de la misma edad (Cavelier 1995), en tanto que a 2400-2500 m.s.n.m. en un promedio de cuatro parcelas de $1250 \mathrm{~m}^{2}$, se encontraron 47 especies en la plantación y 66 especies en el bosque de regeneración natural (Murcia 1997). Estos datos sugieren que las plantaciones de alisos se comportan más como un "inhibidor" de la regeneración que como un "catalizador" (Murcia 1997). De las 16 especies nativas encontradas en el sotobosque de la plantación de alisos de 24-26 años y 2750 m.s.n.m., sólo tres (Verbesina nudipes, Bocconia frutescens y Cestrum humboldtii) son comunes a las 26 especies encontradas bajo el sotobosque de la plantación de alisos de 20 años de edad y 3100 m.s.n.m. (Cavelier 1995). Estas tres especies arbustivas o árboles pequeños típicos de sitios perturbados, parecen tener una amplia distribución en bosques montanos, como lo sugiere la presencia de $V$. nudipes en un bosque montano bajo a 2000 m.s.n.m. en el mismo flanco de la Cordillera Central colombiana (Cavelier \& Tobler 1998).

En las plantaciones abandonadas de $P$. radiata y $C$. lusitanica sólo se encontraron un total cuatro especies leñosas de plantas nativas.
En estas plantaciones se encontraron un número sustancialmente menor de especies con relación al bosque de regeneración natural y a las plantaciones de $A$. acuminata y E. globulus. Esta diferencia podría ser el resultado de que las semillas no están llegando a estos dos tipos de vegetación, o a que las semillas que llegan no logran germinar o que aquellas que germinan no logran establecerse por factores edáficos y microclimáticos. De los factores estructurales medidos en este trabajo, la acumulación de acículas en el suelo de las plantaciones de pinos y cipreses y la alta biomasa de raíces en el suelo de la plantación de cipreses, podrían inhibir el establecimiento de plántulas de especies nativas. El bajo número de especies nativas encontradas en el sotobosque de las plantaciones de pinos y cipreses contrasta con la regeneración natural en plantaciones de estas dos especies a 2000 m.s.n.m. (Cavelier \& Tobler 1998). A esta altitud se encontraron 26 especies de plantas nativas en el sotobosque de la plantación de pinos y 18 especies en el sotobosque de la plantación de cipreses. Estas diferencias podrían explicarse, por lo menos parcialmente, porque en las plantaciones a 2000 m.s.n.m. la densidad de árboles plantados es menor y además no se acumulan acículas debido a una mayor temperatura y precipitación que en las plantaciones a 2750 m.s.n.m. en La Picota (Cavelier $\&$ Tobler 1998).

Una de las especies que logra regenerar bajo las condiciones del sotobosque de $P$. radiata es Ceroxylon quindiuense (Arecaceae), el árbol nacional de Colombia (Madriñan \& Schultes 1995). A pesar de que sólo un individuo de esta especie entró en los censos por tener el diámetro mínimo, otros individuos estaban presentes en el sotobosque de esta plantación. Al parecer, las semillas de esta palma están en capacidad de germinar y establecerse bajo la sombra de pinos, a pesar de la acumulación de acículas en el suelo. Es sabido que esta especie de palma produce gran cantidad de semillas en potreros abiertos, pero estas no germinan debido a las desfavorables condiciones de luz y humedad (Moore \& Anderson 1976, Bernal 1989). 
A nivel estructural, una de las diferencias más notables entre las plantaciones de coníferas y los bosques de regeneración natural es la acumulación de biomasa aérea (Lugo 1992b). Las plantaciones de pinos y cipreses tienen valores de área basal y altura del dosel significativamente mayores que en el bosque de regeneración natural. En contraste, la densidad es mayor en el bosque de regeneración.

Suelos, raíces y hojarasca: No se encontraron diferencias consistentes en las propiedades físicas (textura) y químicas medidas en los suelos orgánico-minerales (Horizonte A), entre plantaciones y bosque de regeneración natural. Mas aún, no se presentaron diferencias consistentes entre los suelos de las plantaciones de coníferas y las de E. globulus o el bosque de regeneración. En contraste, se midieron grandes diferencias en los horizontes orgánicos (L) con acumulaciones importantes de acículas en las plantaciones de las dos coníferas y de raíces en la plantación de ciprés (Cuadro 1). Este resultado está de acuerdo con estudios comparativos entre los suelos de plantaciones de coníferas y bosques nativos, donde los mayores efectos se observan en los horizontes orgánicos (Hofstede 1997, León 1997).

En resumen, las plantaciones abandonadas tanto de especies exóticas (P. radiata, $C$. lusitanica y E. globulus) como nativas (A. acuminata), presentan una regeneración natural que tiene una riqueza de especies sustancialmente menor que la de bosque de regeneración natural producto del abandono de potreros. En los suelos, el efecto es principalmente sobre los horizontes orgánicos debido a la acumulación de acículas y/o raíces finas. La comparación con otros estudios similares sugiere que estos efectos ambientales no son sólo el producto de la especie plantada, sino una combinación de factores que incluyen variables climáticas (temperatura y precipitación) y silviculturales, como la densidad de siembra, podas y entresacas.

\section{AGRADECIMIENTOS}

Agradecemos a Aureliano Sabogal y a los demás miembros de la Corporación Regional del Quíndio (CRQ) por ser apoyo logístico durante la realización de este trabajo. Agradecemos la colaboración de Robert Andersson, Juan Posada, Sergio Llano, Adriana Ruiz, Catalina Sarabia y Catalina Estrada durante el trabajo de campo.

\section{RESUMEN}

Se realizaron censos de vegetación durante 1994 en una parcela de 0.1 ha. en cada una de las plantaciones abandonadas de Pinus radiata, Cupressus lusitanica, Eucalyptus globulus, Alnus acuminata y en un bosque secundario montano alto en los Andes centrales de Colombia. También fueron recolectados datos de las propiedades físicas y químicas de suelos superficiales. El bosque de regeneración natural tiene el mayor número de especies (33) seguido por las plantaciones de E. globulus (26) y A. acuminata (16). Las plantaciones abandonadas de $P$. radiata y C. lusitanica, tuvieron sólo tres especies. Entre el bosque de regeneración natural y la plantación de E. globulus hubo solamente once especies en común y solamente siete con la plantación de A. acuminata. Todas estas especies, con excepción de tres, son arbustos del sotobosque dispersados por viento o aves. En el sotobosque de la plantación de $P$. radiata había una regeneración abundante del árbol nacional de Colombia, Ceroxylum quindiuensis. La altura de los árboles y el área basal fueron significativamente mayores en las plantaciones de $P$. radiata y $C$. lusitanica que en el bosque de regeneración natural. De las variables ambientales y biológicas medidas en este estudio, la acumulación de acículas bajo las plantaciones de $P$. radiata y C. lusitanica y la alta biomasa de raíces finas bajo la plantación de $C$. lusitanica, podrían ser los principales factores limitantes para el establecimiento de un número mayor de especies del bosque nativo. Las propiedades químicas de los suelos varían ampliamente y no hubo diferencias coherentes entre los suelos bajo especies exóticas y nativas.

\section{REFERENCIAS}

Bedoya, E. \& L. Klein. 1996. Forty years of political ecology in the Peruvian upper forest: the case of upper Huallaga, p. 165-186. In L. E. Sponsel, T. N. Headland \& R. C. Bailey (ed.). Tropical deforestation. The human dimension. Columbia University, Nueva York. 
Bello, A. \& L. Katib. 1995. Aspectos ecológicos en la sucesión secundaria de la vegetación subandina (2010$2510 \mathrm{~m}$ ) en localidades del Municipio de Santa Rosa de Cabal, Risaralda. Tesis de Pregrado, Universidad Nacional de Colombia, Bogotá, Colombia.

Bernal, R. G. 1989. Endangerment of Colombian Palms. Principes 33: 113-128.

Brown, S. \& A.E. Lugo. 1990. Tropical secondary forests. J. Trop.Ecol. 6: 1-32.

Cavelier, J. 1992. Fine-root biomass and soil properties in a semideciduous and a lower montane rain forest in Panama. Plant Soil 142: 187-201.

Cavelier, J. 1995. Reforestation with the native tree Alnus acuminata: effects on phytodiversity and species richness in an upper montane rain forest area of Colombia, p. 125-137. In L.S. Hamilton, J.O. Juvik \& F.N. Scatena (eds.). Tropical montane cloud forest. Ecological studies 110. Springer Verlag, Nueva York.

Cavelier, J. \& A. Etter. 1995. Deforestation of montane forest in Colombia as a result of illegal plantations of opium (Papaver somniferum), p.125-137. In S. P. Churchill, H. Balslev, E. Forero \& J. L. Luteyn (eds.). Biodiversity and conservation of neotropical montane forest. The Nueva York Botanical Garden, Bronx, Nueva York.

Cavelier, J \& A. Tobler. 1998. The effect of abandoned plantations of Pinus radiata and Cupressus lusitanica on soils and regeneration of a tropical montane rain forest in Colombia. Biodiversity Conserv. 7: 335-347.

Clinebell II, R.R., O.L. Phillips, A.H. Gentry, N. Stark \& H. Zuuring. 1995. Prediction of neotropical tree and liana species richness from soil and climatic data. Biodiversity Conserv. 4: 56-90.

Ewel, J. 1980. Tropical succession. Mainfold routes to maturity. Biotropica 12: 2-7.

Gentry, A.H. 1988. Changes in plant community diversity and floristic composition on environmental and geographical gradients. Ann. Miss. Bot. Gard. 75: 1-34.

Gentry, A.H. 1992. Diversity and floristic composition of Andean forests of Penu and adjacent countries: implication for their conservation. Mem. Mus. Hist. Nat. (Lima) 21: 11-29.

Henderson, A., S.P. Churchill \& J.L. Luteyn. 1991. Neotropical Plant Diversity. Nature 351: 21-2.

Hofstede, R. 1997. El impacto ambiental de plantaciones de Pinus en la Sierra del Ecuador. Proyecto Ecopar, Quito. 53 p.
Kelliher, F. M., R. Leuning \& E. D. Schultze. 1993. Evaporation and canopy characteristics of coniferous forests and grasslands. Oecologia 95: 153-163

Landon, J. R. 1991. Booker tropical soil manual. Longman, Essex, Inglaterra. 474 p.

León, T. 1997. Efectos sobre el suelo de plantaciones comerciales de Pinus patula y E. grandis en crecimiento. Corporación Nacional de Investigación y Fomento Forestal, CONIF, Bogotá, Colombia. $51 \mathrm{p}$.

Lima, W. de P. 1987. O reflorestamento com Eucalipto e seus impactos ambientais. Artpress, São Paulo. 114 p.

Londoño, E. 1994. Parque Regional Natural Ucumari. Un vistazo histórico, p. 13-24. In O. Rangel (ed.). Ucumarí: un caso típico de la diversidad biótica andina. Corporación Autónoma Regional de Risaralda. CARDER. Pereira, Risaralda, Colombia.

Lugo, A. E. 1992a. Tree plantations for rehabilitating damaged Forest Lands in the tropics, p. 247-255. In M. K. Balli (ed.). Ecosystem rehabilitation, vol. 2: ecosystem analysis and synthesis. SPB, La Haya.

Lugo, A. E. 1992b. Comparison of tropical tree plantations with secondary forest of similar age. Ecol. Monogr. 62: 1-41.

Madriñan, S. \& R. E. Schultes. 1995. Colombian's national tree: the wax palm Ceroxylon quindiuense and its relatives. Elais 7: 35-56.

Molano, J. 1992. Las regiones tropicales americanas: visión geográfica de James J. Parsons. Fondo FEN, Bogotá, Colombia. 279 p.

Moore, H. E. Jr. \& A. B. Anderson. 1976. Ceroxylon alpinum and Ceroxylon quindiuense (Palmae). Gentes Herbarum 11: 168-185.

Murcia, C. 1997. Evaluation of Andean alder as a catalyst for the recovery of tropical cloud forests in Colombia. For. Ecol. Manag. 99: 163-170.

Parrota, J. A. 1992. The role of plantations forests in rehabilitating degraded tropical ecosystems. Agri. Ecosyst. Environ. 41: 115-133.

Savill, P.S. \& J. Evans. 1986. Plantation sylviculture in temperate regions: with special reference to the British Isles. Clarendon, Oxford.

Sawyer, J. 1993. Plantations in the tropics. IUCN, Gland, Suiza. 83 p. 
Stiles, G. \& L. Rosselli. 1993. Consumption of fruits of the Melastomataceae by birds: how diffuse is coevolution? Vegetatio 107/108: 57-73.

Viña, A. \& J. Cavelier. 1999. Deforestation rates (1938-1988) of tropical lowland forests on the Andean foothills of Colombia. Biotropica (en prensa).
Waterloo, M. J. 1994. Water and nutrient dynamics of $P i$ nus caribaea plantation forest on former grassland soils in southwest Viti Levu, Fidji. Febodruk B. V., Enschede. 478 p.

Young, K. 1996. Threats to biological diversity caused by coca/cocaine deforestation in Perú. Environ. Conserv. 23: 7-15. 


\section{ANEXO 1}

Composición florística de las plantaciones abandonadas y del bosque de regeneración natural. Para cada tipo de vegetación se indica el número de individuos por especie presentes en una parcela de 0.1 ha.

\begin{tabular}{|c|c|c|c|c|c|c|}
\hline Familia & Especie & Pino & Ciprés & Aliso & Eucalipto & R. natural \\
\hline Acanthaceae & Aphelandra acanthus Nees & & & & & 1 \\
\hline \multirow[t]{8}{*}{ Asteraceae } & Asploddianthus pseudostuebelli King\& $\mathrm{H}$. & & & 5 & & 5 \\
\hline & Baccharis latifolia (R.\&P.) Peersoon & & & & 3 & 11 \\
\hline & Barnadesia pirviflora Spruce ex Benth. & & & 26 & 3 & \\
\hline & Barnadesia spinosa L.f. & & & & 1 & \\
\hline & Iochroma gesnerioides (Kunth)Miers & & & & 1 & \\
\hline & Joseanthus trichotomus (Gleas) H.Rob. & & & 1 & & \\
\hline & Schistocarpha eupatorioides Hieron & & & 1 & 41 & \\
\hline & Verbesina nudipes Blake & & 2 & 2 & 1 & 2 \\
\hline Arecaceae & Ceroxylon quindiuense $\mathrm{H}$. Wendl. & 1 & & 1 & & \\
\hline Betulaceae & Alnus acuminata Kunth & & 6 & 50 & 1 & \\
\hline \multirow[t]{3}{*}{ Boraginaceae } & Cordia cylindrostachya (Ruiz\& Pav.) & & & & & \\
\hline & Roem. \& Schult. & & & 1 & 1 & \\
\hline & Tournefortia fuliginosa Kunth & & & & & 1 \\
\hline Brunelliaceae & Brunellia sp. & & & & 1 & \\
\hline Buddlejaceae & Buddleja bullata Kunth & & & & 1 & \\
\hline Caricaceae & Carica pubescens Lenné \& Koch & & & 2 & & \\
\hline Chloranthaceae & Hedyosmum bonplandianumKunth & & & & & 3 \\
\hline Cupressaceae & Cupressus lusitanica Mill. & . & 130 & & & \\
\hline Cyatheaceae & Sphaeropteris quindiuensis (Karst.) Tyron & & & & 1 & 1 \\
\hline Flacourtiaceae & Abatia parviflora Ruiz \& Pav. & & & 1 & & 3 \\
\hline Heliantheae & Clibadium aff pacificum Cuatrec. & & & & & 1 \\
\hline \multirow[t]{7}{*}{ Melastomataceae } & Axinaea macrophyla (Naudin) Triana & & & & & 16 \\
\hline & Leandra melanodesma (Naudin) Cogn & & & 3 & 10 & 6 \\
\hline & Melasto 1 & & & & & 2 \\
\hline & Miconia pustulata Naudin & & & & & 67 \\
\hline & Miconia theaezans (Bonpl.) Cogn. & & & & 1 & \\
\hline & Tibouchina lepidota (Bonpl.) Baill. & & & & & 2 \\
\hline & Tibouchina mollis (Bonpl.) Cogn. & & & & 1 & I \\
\hline \multirow[t]{2}{*}{ Myrtaceae } & Eucalyptus globulus Labill & & & & 94 & \\
\hline & Myrcianthes leucoxyla (Ort.) Mc. Vaugh & 1 & & & & \\
\hline Pappaveraceae & Bocconia frutescensL. & & & 3 & & 1 \\
\hline \multirow[t]{3}{*}{ Pinaceae } & Pinus patula (Schide \& Deppe ex) & & & & & \\
\hline & Schult. \& Chosu. & & & 1 & & \\
\hline & Pinus radiata $\mathrm{D}$. Don & 91 & & & & \\
\hline Piperaceae & Piper lacunosumKunth & & & & 1 & \\
\hline Polygalaceae & Monnina angustataTriana \& Planch. & & & & 1 & 2 \\
\hline Rubiaceae & Palicourea sp. & & & & & 2 \\
\hline Sabiaceae & Meliosma arenosa Idrobo \& Cuatrec. & & & & & 1 \\
\hline Sapindaceae & Allophylus mollis(Kunth) Radlk. & & & & 1 & \\
\hline Saxifragaceae & Escallonia paniculata (R.\& P.) Roem \& Sc & & & & & 1 \\
\hline \multirow[t]{10}{*}{ Solanaceae } & Cestrum humboldtiiFrancey & & & 1 & & \\
\hline & Dunalia solanacea Kunth & & & & 2 & 5 \\
\hline & Lycianthes radiata (Sendth.) Bitter & & & & 1 & \\
\hline & Sessea corymbosa Miers & & & & & 9 \\
\hline & Sessea crassivenosa Bitter & & & 2 & 2 & $\cdot$ \\
\hline & Solanum aff. scorpioideum Rusby & $\theta$ & & 7 & 1 & 2 \\
\hline & Solanum amblophyllumHook & & & & 2 & \\
\hline & Solanum aphydendronS. Knapp. & & & & 4 & 1 \\
\hline & Solanum hypoleurotrichum Bitter & & & & & 1 \\
\hline & Solanum ovalifolium Dunal & & & & & 3 \\
\hline Theaceae & Freziera canescens Bonpland & & & & 1 & \\
\hline \multirow[t]{6}{*}{ Verbenaceae } & Lippia hirsuta L.f. & & & 1 & 2 & 9 \\
\hline & Indet 1 & & & & & 1 \\
\hline & Indet 2 & & & & & 8 \\
\hline & Indet 3 & & & & & 1 \\
\hline & Indet 4 & & & & & 1 \\
\hline & Indet 5 & & & & & 1 \\
\hline
\end{tabular}

